\title{
An adaptive Second Level Hybrid Image Watermarking Technique using DWT-SVD in Low Frequency Band
}

\author{
Rajesh Tyagi ${ }^{1}$, M. K. Pandey ${ }^{2}$ \\ Research Scholar, Dept of Electronics and Communication Engineering, RJIT, Gwalior, India ${ }^{1}$ \\ Assistant Professor, Dept of Electronics and Communication Engineering, RJIT, Gwalior, India ${ }^{2}$
}

\begin{abstract}
Day by day the increase in technology and other multimedia systems has created an urgent need for copyright ownership of multimedia contents. Digital Image Watermarking is one such technology that has been developed to protect digital images from illegal manipulations. To achieve this goal, here we present a hybrid image watermarking algorithm based on Discrete Wavelet Transforms and Singular Value Decomposition to protect the watermark image information or hide such that assumption made like visible image is cover image and other is watermark image for content ownership. The singular value is going to embedded with watermark singular values making use of scaling factor $(\alpha)$. The experimental results show the effectiveness of hybrid image watermarking scheme. Performance of methodology is evaluated using different fidelity parameters like as peak signal noise ratio (PSNR) and normalized cross correlation (NCC).
\end{abstract}

Keywords: Image watermarking, discrete wavelet transforms, SVD, PSNR; CC.

\section{INTRODUCTION}

In present digital era, the ubiquitous network environment has promoted the rapid delivery of digital multimedia data. Users are eager to enjoy the convenience and advantages that networks have provided. Meanwhile, users are eager to share various media information in a rather cheap way without awareness of possibly violating copyrights. In view of these several hiding techniques are available.

Data hiding is a very active research subject of the areas of information security and multimedia signal. Due to development of science, now day's human mostly depend upon computer in different area. Therefore, authentication, information security and other various issues are raised with multimedia sources and content. Digital data can be stored efficiently and with a very high quality, and it can be manipulated very easily using Computers. These information, which include still images, video, audio, or text are stored and transmitted in a digital format. Information stored in digital format can be easily copied without loss of quality and efficiently distributed [1]. The digital watermark is then introduced to solve this problem. Digital watermarking is a branch of information hiding which is used to hide proprietary information in digital media like photographs, digital music, or digital video [23]. The ease with which digital content can be exchanged over the Internet has created copyright Infringement issues. Copyrighted material can be easily exchanged over peer-to-peer networks, and this has caused major concerns to those content providers who produce these digital contents.

Generally, the image watermarking can be done in spatial domain or in transform domain [6]. Compared to spatial domain techniques [4].frequency-domain watermarking techniques proved to be more effective with respect to achieving the imperceptibility and robustness requirements of digital watermarking algorithms [5]. Commonly used frequency-domain transforms include the Discrete Wavelet Transform (DWT), the Discrete Cosine Transform (DCT) and Discrete Fourier Transform (DFT). However, DWT has been used in digital image watermarking more frequently due to its excellent spatial localization and multi-resolution characteristics, which are similar to the theoretical models of the human visual system. Further performance improvements in DWTbased digital image watermarking algorithms could be obtained by increasing the level of DWT.

The quality of watermarked image can be determined based on some important factors given like as: Firstly, in imperceptibility the quality of original image must not be changed due to the watermark [5] [6]. Secondly, robustness of image, in this the watermark removal is difficult in case of different types of attacks like noise addition, compression, scaling and rotation etc. [7] [8]. Thirdly, in capacity the most information is embedded in spatial domain as well as in transformation domain. But there are some disadvantages in both spatial and transformation domain like in spatial domain it is not much robust against image processing attacks.

\section{DISCRETE WAVELET TRANSFORMS (DWT)}

Discrete Wavelet transform (DWT) is a mathematical tool for hierarchically decomposing an image. The transform 
of a signal is just another form of representing the signal. It does not change the information content present in the signalIt is useful for processing of non-stationary signals. Wavelet transform provides both frequency and spatial description of an image. Unlike conventional Fourier transform, temporal information is retained in this transformation process. Wavelets are created by translations and dilations of a fixed function called mother wavelet. Wavelet can represent a signal in time-frequency domain. Analyzing a signal with this kind of representation gives more information about the when and where of different frequency components. In other words, wavelet transform not only transforms the time domain representation of a signal to the frequency domain representation, but also preserves spatial information in the transform. This feature enhances the image quality especially for the low bit rate representation. The DWT is a multi-resolution technique that can analyze different frequencies by different resolutions. This section analyses suitability of DWT for image watermarking and gives advantages of using DWT as against other transforms. For 2-D images, applying DWT corresponds to processing the image by 2 -D filters in each dimension. The filters divide the input image into four no overlapping multi-resolution sub-bands LL1, LH1, HL1 and HH1. The sub-band LL1 represents the coarse-scale DWT coefficients while the sub-bands LH1, HL1 and HH1 represent the fine-scale of DWT coefficients. Due to its excellent spatial-frequency localization properties, the DWT is very suitable to identify the areas in the host image where a watermark can be embedded effectively. In general most of the image energy is concentrated at the lower frequency sub-bands LLx and therefore embedding watermarks in these subbands may degrade the image significantly. Embedding in the low frequency sub-bands, however, could increase robustness significantly. On the other hand, the high frequency sub bands HHx include the edges and textures of the image and the human eye is not generally sensitive to changes in such sub-bands. This allows the watermark to be embedded without being perceived by the human eye.

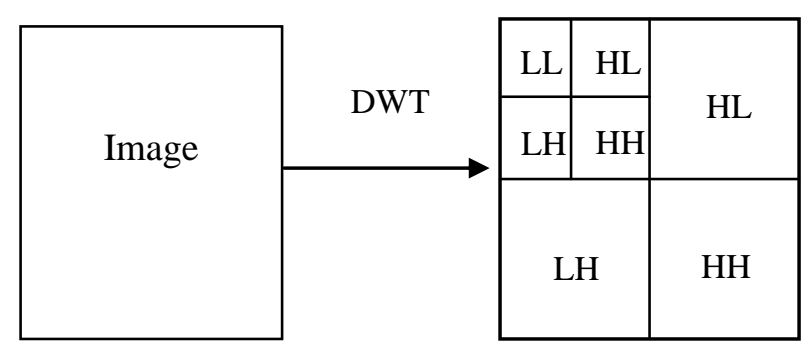

Fig.1 showDWT decomposition of image at level-2

As pointed out, wavelet-based watermarking methods exploit the frequency information and spatial information of the transformed data in multiple resolutions to gain robustness. Wavelet Transform is computationally efficient and can be implemented by using simple filter. Magnitude of DWT coefficients is larger in the lowest bands (LL) at each level of decomposition and is smaller for other bands (HH, LH and HL). The larger the magnitude of the wavelet coefficient the more significant it is. Watermark detection at lower resolutions is computationally effective because at every successive resolution level there are few frequency bands involved. High resolution sub bands helps to easily locate edge and textures patterns in an image.

Wavelet transforms use wavelet filters to transform the image. There are many available filters, although the most commonly used filters for watermarking are:- Haar Wavelet Filter, Daubechies Orthogonal Filters, Daubechies Bi-Orthogonal Filters, Symlet wavelets. Each of the filters decomposes the image into several frequencies. Single level decomposition gives four frequency representations of the images.

\section{OVERVIEW OF SINGULAR VALUE DECOMPOSITION (SVD)}

From the discernment of image processing an image can be viewed as a matrix with nonnegative scalar entries. SVD is an effective numerical analysis tool from linear algebra to decompose a rectangular matrix "A" into an orthogonal matrix $\mathrm{U}$, diagonal matrix $\mathrm{S}$, and the transpose of an orthogonal matrix $\mathrm{V}[3,4,7,10]$. SVD decomposes a given image $\mathrm{A}$ of size $\mathrm{M} \times \mathrm{N}$ as

$$
\mathrm{A}=\mathrm{USVT}
$$

$\mathrm{U}$ and $\mathrm{V}$ are orthogonal matrices of size MxM and NxN, respectively. $\mathrm{S}$ is a diagonal matrix of size MxN having singular values and satisfy the property $\sigma 1 ; 1>\sigma 2 ; 2>\sigma 3 ; 3$ $>\ldots \ldots>\sigma n ; n$ : It is worth noting that, the singular vectors of an image specify the image "geometry" similarly left singular vectors represent horizontal details and right singular vectors represent the vertical details of an image, while the singular values specify the "luminance" (energy) of the image. Slight variations in the singular values do not affect the visual perception of the quality of the image. Watermark embedding through slight variations of singular values in the segmented image has been introduced as a choice for robust watermarking through minor modification of the singular values of the original image

\section{WATERMARKING TECHNIQUE}

Here, we propose an adaptive second level hybrid image Watermarking Technique using DWT-SVD in low frequency band. The proposed algorithm is divided into two parts, watermark embedding and watermark extraction.

a) Watermark Embedding

The watermark embedding process is described below as following:

Step.1: Load the cover image and watermark image.

Step.2: Decomposed both the image into four sub-bands using DWT for cover and watermark images respectively.

Step.3: after taking DWT, we decomposed both the image using SVD. 
Step.4: Compute new sigma matrix using fusion of both sigma matrix.

Step.5: Using new computed signal matrix Snew, New LL band is computed with inverse SVD.

Step.5: Therefore, watermarked image obtained using inverse DWT based on LLnew band and remaining sub band of cover image.

\section{B. Watermark Extraction}

Watermark extraction process is also very important process, it give the hidden information from watermarked image; which are embedded into cover image. The watermark embedding process is described below as following and shown in fig. 3.3:

Step.1: Load the cover image, watermark image and watermarked image.

Step.2: Decomposed the images into sub-bands using DWT respectively.

Step.3: after taking DWT, we decomposed images using SVD

Step.4: Compute new sigma matrix using fusion of both sigma matrix using scaling factor work as key in watermark embedding process.

Step.5: Using new computed signal matrix Snew, New LL band is computed with inverse SVD.

Step.5: Therefore, extracted watermark image obtained using inverse DWT based on LLnew band and reaming subband.

\section{RESULTS AND DISCUSSION}

Over all analysis has done with 512X512 image and evaluated with consider fidelity parameters.Here, images used which are obtained from USC-SIPI image database as a standard evaluation database for watermarking algorithms.Here, results analysis of proposed technique illustrate the efficiency of proposed watermarking technique

\section{EVALUATION FIDELITY PARAMETERS}

The visual performance of watermarked images is determined by using peak signal-to-noise ratio (PSNR) and Normalized Correlation which are historically adopted in image processing in order to evaluate the performance of the output results as shown in tables.

$$
\begin{aligned}
& M S E=\frac{1}{N M} \sum_{i=1}^{N} \sum_{j=1}^{M}(f(i, j)-g(i, j)) \\
& P S N R=10 \log _{10} \frac{L^{2}}{M S E}
\end{aligned}
$$

From eq. (1), L shows the values of pixel range. As MSE is inversely proportional to PSNR, thus the small mean square error tends to high signal to noise ratio. The quality measurement for image is directly measure from the pixel values. For better image quality the PSNR must be high. The quality of the image is measured using normalized cross co-relation (NCC) and is obtained by using eq. (2)

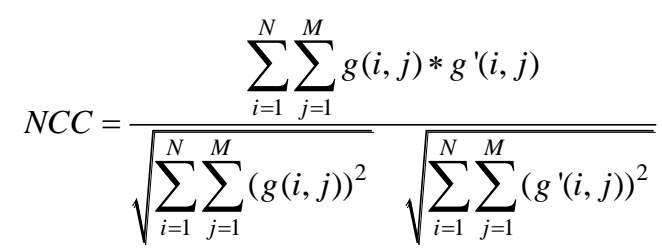

Here, PSNR considered for good efficiency is close to 35 $\mathrm{dB}$ to avoid having a visible watermark but at the same time including the watermark with a large energy to be resistant to attacks.

\section{SIMULATED EXPERIMENTAL RESULTS}

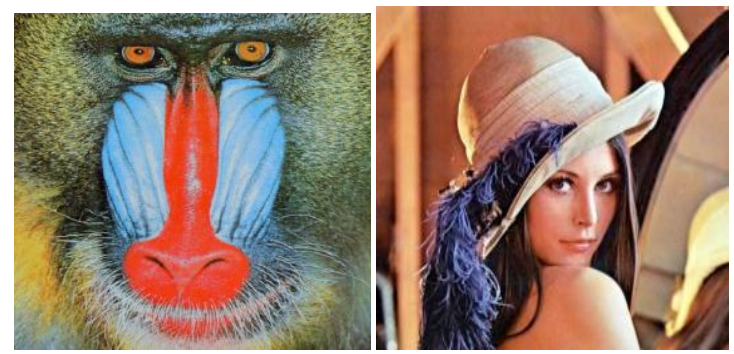

Figure 3(a): ( mandrill and Lena), size (512x512)).

\section{Sample of watermark image}

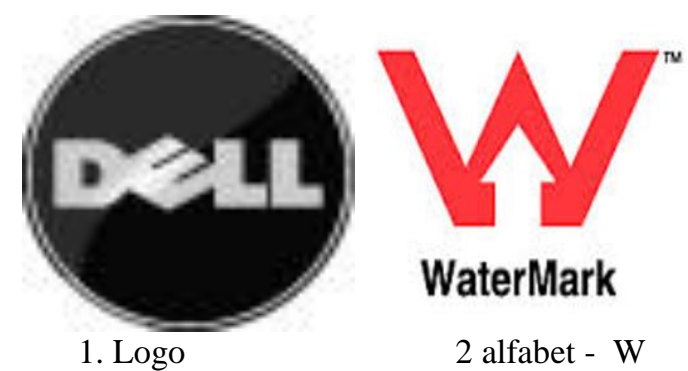

Figure 3(b): watermark image

Fig. 3(a) shows the original image and Fig. 3(b) shows the watermark image for embedding of watermark in the original image

Table I shows the PSNR Value and NCC value for 2 levels of DWT-SVDbased image watermarking techniques (mandrill (as cover) \&Dell- logo (as watermark)

\begin{tabular}{|c|l|l|}
\hline Visibility factor & \multicolumn{2}{|c|}{ Watermarked image } \\
\hline & PSNR & NCC \\
\hline 0.01 & 45.09 & 0.9978 \\
\hline 0.02 & 39.06 & 0.9994 \\
\hline 0.03 & 35.58 & 0.9997 \\
\hline 0.05 & 31.15 & 0.9999 \\
\hline 0.1 & 25.14 & 1.0 \\
\hline 1 & 8.81 & 0.9734 \\
\hline
\end{tabular}

Bar chart show the value of PSNR at different value of scaling factor.

As seen in simulated results, here we proposed 2-level DWT-SVD technique. Here we have used images mandrill as original image and the Dell-logo image as the watermark. 


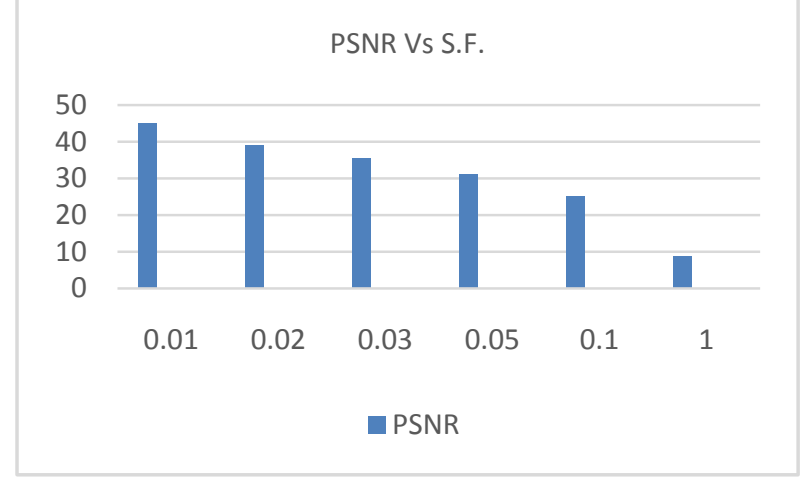

Both the images are of equal size of 512X512.The value of visibility factor $\mathrm{k}$ is varied from 0.01 to 0.1 and we see that as we decrease the value of visibility factor, the value of PSNR increases but the same time the value of CC decreases hence to get the best result we set the value of visibility factor at 0.02 .
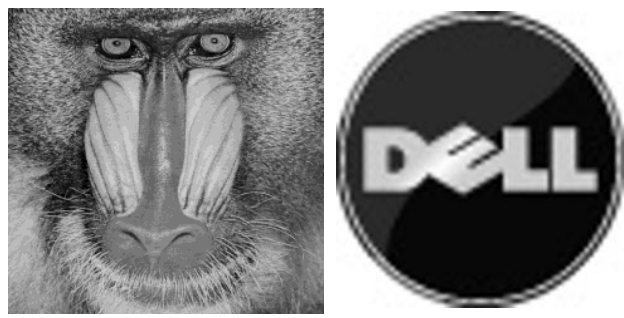

Watermarked image\& recover water mark image at 0.02using 2-Level dwt

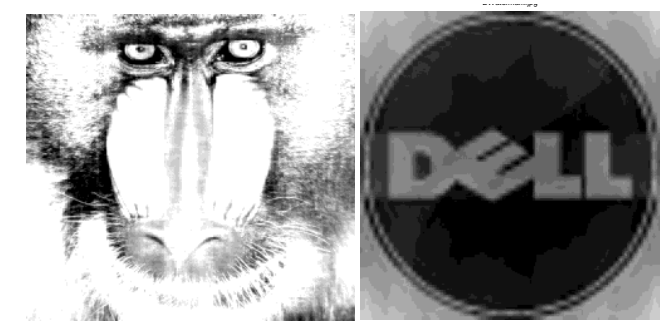

Watermarked image\& recover water mark image at 1.0 using 2-Level dwt
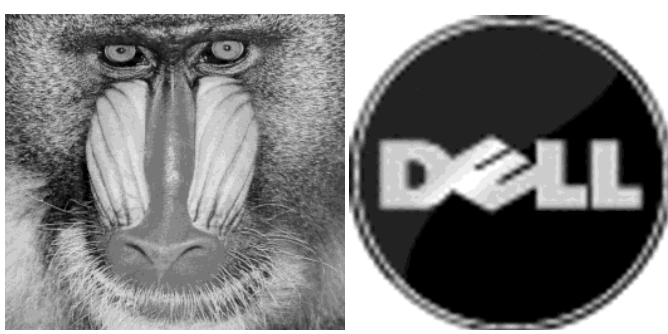

Watermarked image\& recover water mark image at 0.1 using $21 \mathrm{dwt}$

Fig. 4 shows the best result of watermarked image and recover watermark image at 0.02

The simulated experimental results also evaluated with visual representation of watermarked and extracted watermark image for human vision system (HVS). Results are clearly seen that the proposed methodology having robust efficiency of watermarking with data hiding ability. All experiments are shown the proposed algorithm is efficient in data hiding properties as per HVS.

\section{CONCLUSION}

In this paper, an image watermarking technique based on a 2-level discrete wavelet transform has been presented and implemented. This technique can embed the invisible watermark into salient features of the image using variable visibility factor. Experiment results shows that the quality of the watermarked image and the recovered watermark are dependent only on the visibility factors and also indicate that the 2- level DWT provide better performance. All the results obtained for the recovered images and the watermark are identical to the original images. Results are clearly seen that the proposed methodology having robust efficiency of watermarking with data hiding ability.

\section{REFERENCES}

[1] W. Bender, D. Gruhl, N. Morimoto and A. Lu, "Techniques for Data Hiding," IBM System Journal, vol. 35, NOS 3\&4, pp. 313-336, 1996.

[2] E. T. Lin and E. J. Delp, "A Review of Data Hiding in Digital Images," in Proc. of the Image Processing, Image Quality, Image Capture Systems Conf. (PICS' 99), pp. 274-278, Apr. 1999.

[3] P. H. W. Wong, O. C. Au and G. Y. M. Yeung, "A Novel Blind Multiple Watermarking Technique for Images," accepted by IEEE Transactions on Circuits and Systems for Video Technology: Special Issue on Authentication, Copyright Protection and Information Hiding, Sept. 2003

[4] Mr. Gaurav N Mehta, Mr. Yash Kshirsagar, Mr. Amish Tankariya,“ Digital Image Watermarking: A Review”, International Journal of Scientific Engineering and Technology (ISSN : 2277-1581) www.ijset.com, Volume No.1, Issue No.2 pg:169-174 01 April 2012

[5] Singh A. P., Mishra A., Wavelet Based Watermarking on Digital Image, Indian Journal of computer Science and Engineering 2011

[6] L. Hu, F. Wan, 'Analysis on wavelet coefficient for image watermarking', Int. Conf. MINES'10, pp. 630-634, 2010

[7] Ezz El-Din Hemdan et al. "Hybrid Digital Image Watermarking Technique for Data Hiding" IEEE 30th national radio science conference 2013, pp220-227, April 2013

[8] Samira Lagzian, Mohsen Soryani, Mahmood Fathy, 'A New Robust Watermarking Scheme Based on RDWT-SVD'International Journal of Intelligent Information Processing, Vol. 2, Number 1, 2011, Doi:10.4156/ijiip.vol2. issue1.3

[9] Komal Tomar, "A Review Paper of Different Techniques on Digital Image Watermarking Scheme For Robustness" International Journal of Advanced Research in Computer Science and Software Engineering,volume 5,Issue 2, pp 900-904,February 2015

[10] Dr. J. Abdul Jaleel, Jisha Mary Thomas : "Guarding Images Using A Symmetric Key Cryptographic Technique: Blowfish Algorithm“, ISSN: 2277-3754 ISO 9001:2008 Certified International Journal of Engineering and Innovative Technology (IJEIT) Volume 3, Issue 2, August 2013

[11] Ansu Anna Ponnachen, Lidiya Xavier, "Dwt Based Watermarking For Lifting Based Compression And Symmetric Encryption Of Jpeg Images", International Journal of Engineering Research \& Technology (IJERT) ISSN: 2278-0181 www.ijert.org Vol. 2 Issue 6, June - 2013

[12] Ghazali Bin Sulong, Harith Hasan(Corresponding author), Ali Selamat, Mohammed Ibrahim and Saparudin, "A New Color Image Watermarking Technique Using Hybrid Domain", IJCSI International Journal of Computer Science Issues, Vol. 9, Issue 6, No 1, November 2012

[13] Akhil Pratap Singh," Wavelet Based Watermarking On Digital Image “, Indian Journal of Computer Science and Engineering Vol No 2 . 